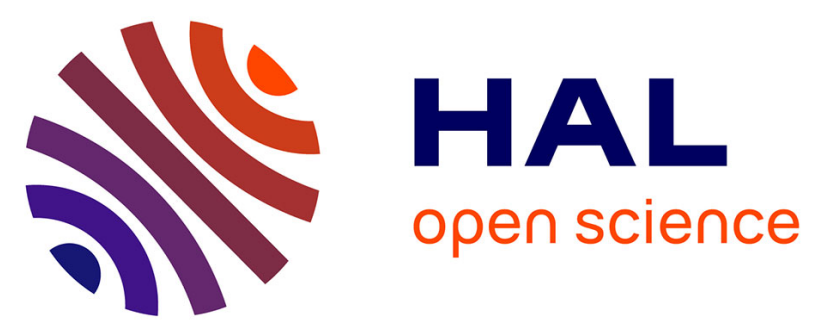

\title{
In situ 3D X-ray microtomography of laser-based powder-bed fusion (L-PBF) - A feasibility study
}

Pierre Lhuissier, Xavier Bataillon, Camille Maestre, Julien Sijobert, Elodie

Cabrol, Philippe Bertrand, Elodie Boller, Alexander Rack, Jean-Jacques Blandin, Luc Salvo, et al.

\section{To cite this version:}

Pierre Lhuissier, Xavier Bataillon, Camille Maestre, Julien Sijobert, Elodie Cabrol, et al.. In situ 3D X-ray microtomography of laser-based powder-bed fusion (L-PBF) - A feasibility study. Additive Manufacturing, In press, 10.1016/j.addma.2020.101271 . hal-02558318

\section{HAL Id: hal-02558318 https://hal.science/hal-02558318}

Submitted on 29 Apr 2020

HAL is a multi-disciplinary open access archive for the deposit and dissemination of scientific research documents, whether they are published or not. The documents may come from teaching and research institutions in France or abroad, or from public or private research centers.
L'archive ouverte pluridisciplinaire HAL, est destinée au dépôt et à la diffusion de documents scientifiques de niveau recherche, publiés ou non, émanant des établissements d'enseignement et de recherche français ou étrangers, des laboratoires publics ou privés. 


\title{
In situ 3D X-ray microtomography of laser-based powder-bed fusion (L-PBF) - A feasibility study
}

Pierre Lhuissier ${ }^{1 \#, ~ X a v i e r ~ B a t a i l l o n ~}{ }^{1}$, Camille Maestre ${ }^{1}$, Julien Sijobert ${ }^{2}$, Elodie Cabrol$^{2}$, Philippe Bertrand $^{2}$, Elodie Boller ${ }^{3}$, Alexander Rack ${ }^{3}$, Jean-Jacques Blandin ${ }^{1}$, Luc Salvo $^{1}$, Guilhem Martin ${ }^{1 \#}$

1. Univ. Grenoble Alpes, CNRS, Grenoble INP, SIMaP, F-38000 Grenoble, France

2. Ecole Nationale d'Ingénieurs de Saint Etienne, Laboratoire de Tribologie et de Dynamique des Systèmes, UMR 5513 CNRS, Univ. Lyon, 58, Rue Jean Parrot, 42023 Saint Etienne, France

3. ESRF-The European Synchrotron, CS40220, 38043, Grenoble Cedex 9, France

\# Corresponding Authors:

Email: pierre.lhuissier@simap.grenoble-inp.fr

Email: guilhem.martin@ simap.grenoble-inp.fr

\begin{abstract}
X-ray microtomography can be used to characterise objects undergoing fabrication by additive manufacturing. During the layer-by-layer building process, it can provide key information about geometry, roughness and it can even reveal typical defects such as lack-offusion porosity, gas pores or cracks. Usually, objects are built with varied processing parameters and then characterised post-mortem. In the present work, we describe our custom-designed additive manufacturing chamber allowing in situ 3D-non-destructive characterisation to be performed during layer-by-layer construction using synchrotron X-ray microtomography. Scans before (subsequently to powder deposition) and after local laser melting are acquired for every layer. A few examples of such a characterisation demonstrate the ability of the set-up to reproduce conditions close to those used in conventional laser powder-bed fusion devices and to reveal key phenomena.
\end{abstract}

Keywords: Powder-bed fusion (PBF); X-ray microtomography; In situ; Synchrotron 


\section{Graphical Abstract}

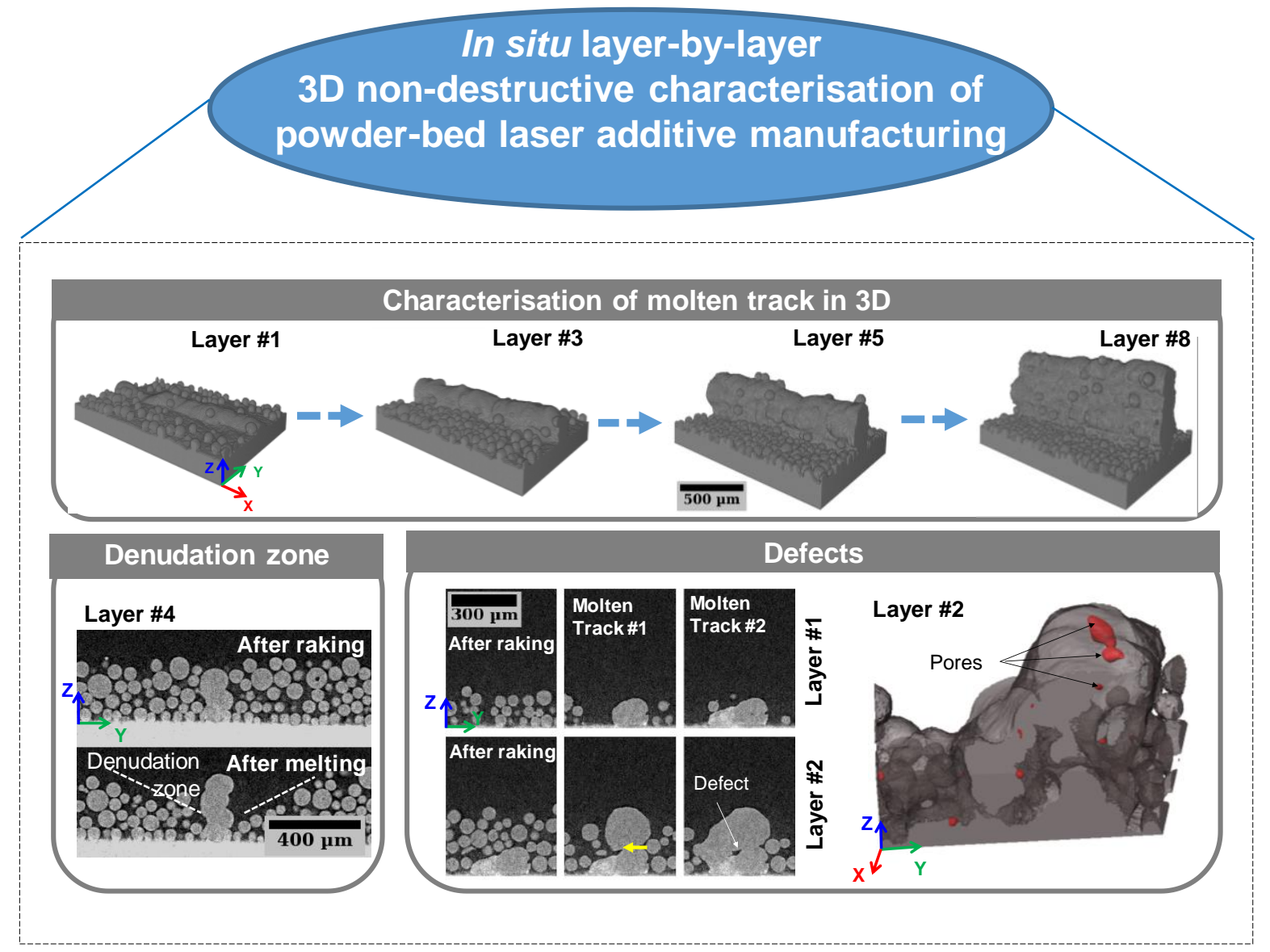




\section{Introduction}

Laser powder-bed fusion (L-PBF) is one of the most popular technologies for additive manufacturing (AM) because it offers the possibility to produce objects with complicated geometry [1,2]. The aerospace and medical industries have been key drivers for the development of this technology [1,3]. The basic principle of laser-based powder-bed fusion additive manufacturing consists of selectively melting a thin layer of powder. This process is repeated to build 3D objects layer-by-layer. However, very complex dynamics and transient phenomena occur when melting a powder bed because of the typical processing conditions such as localised heating, high cooling rates and thermal cycling induced by the layer-by-layer construction process. Laser-matter interactions are believed to strongly affect the melt pool dimensions and dynamics, hence altering the quality of the molten track [4-6] and built objects. Process-induced defects can lead to a drastic reduction in the mechanical properties and quality of an object such as its roughness and geometrical dimensions. The main causes of defects are attributed to powder spattering [7,8] and pore formation [8]. The scientific community has made a tremendous effort to shed light on the underlying mechanisms of additive manufacturing based on process monitoring.

In situ process monitoring of laser-matter interactions have first been carried out using high-speed optical cameras or thermal sensors, see e.g. [7,9-11]. Powder spatter and molten track quality were thus characterised, see e.g. [7,9-11]. However, the use of high-speed optical cameras is limited to powder bed surface observations and does not allow the monitoring of the melt pool evolution during localised melting events. Recently, several research groups have characterised laser-based powder bed fusion additive manufacturing relying on time-resolved $\mathrm{X}$-ray imaging. Significant efforts have been made to design replicators to mimic laser-based powder bed fusion while allowing X-ray synchrotron imaging to be performed, see process replicator details in [12-14]. These process replicators used in combination with high-speed Xray synchrotron radiography have brought new insights into the evolution of melt pool size and morphology during localised melting [15-18], the mechanisms of defect formation such as pores $[15,17-19]$ or powder spatters $[12,15,17,20]$ as well as solidification velocity [15]. Such in situ synchrotron radiation characterisation has been carried out on several materials: Ti6Al4V-alloy [14,15], Invar 36 [12,17], 316L stainless steel [18,20] and AlSi10Mg alloy $[13,16,20]$. Although MHz-frame rate X-ray radiography has revealed the melt pool dynamics and clarified the underlying physical mechanisms, it still suffers from the lack of 3D information at every step of the process. In addition, the literature on these topic has been limited to single tracks in a powder bed width of typically a few hundreds of microns. This can be seen as a situation relatively different compared to AM-processes where parts are made of adjacent molten tracks and stacked layers. Some authors have also used X-ray diffraction in combination with high-speed X-ray radiography to provide information regarding phase transformation [15] and others have run additional post-mortem X-ray microtomography scans to obtain a full 3D description of the final melting tracks $[17,18]$.

The purpose of the present work is to demonstrate the feasibility to extend the in situ characterisation of powder bed fusion AM-processes to 3D. A custom-designed laser-based powder bed fusion instrument allowing 3D-characterisation using X-ray microtomography is described. To our knowledge, this is the first instrument offering the possibility to achieve a full 3D description of powder bed fusion additive manufacturing layer-by-layer over several layers. Our custom-designed instrument allows 3D-builds to be monitored with scans after 
powder deposition and laser melting for each built layer or for any desired event of the process. Contrary to the instruments described in [12-15], our system does not permit laser-matter interactions to be investigated, but rather enables 3D images to be obtained at any step of the layer-by-layer building process. A detailed description of our custom-designed device is given before showing a few illustrative examples of what can be observed and inferred from such experiments. As previously highlighted, AM-processing routes have been developed to produce 3D components made of adjacent and stacked molten tracks. There are defects forming within a single track but a lot of them result from melting adjacent tracks and stacked layers. Thus, the main additional insight provided by our approach is the monitoring of a defect population. For instance, persisting defect or, on the contrary, defect healing can be revealed. Other additional insights can be (i) a full 3D description of the powder bed stacking and packing before melting, and (ii) to overcome artifacts induced by a 2D characterisation. This paper demonstrates the technical feasibility and validates our custom-designed laser-based powder bed fusion instrument adapted for 3D-monitoring of the layer-by-layer building process at beamline ID19 of the European Synchrotron Radiation Facilities (ESRF). Thus, this report provides proof-ofconcept rather than a fundamental understanding of the defect formation during additive manufacturing which was the aim of subsequent investigations.

\section{Materials and Methods}

In the laser powder bed fusion additive manufacturing process, a calibrated layer thickness is first deposited and subsequently melted with a focused laser beam deflected by optical mirrors. The overall process must be conducted in a controlled atmosphere to prevent possible contamination such as oxidation. Such a device consists of two main components: a high energy source (laser) and a build chamber allowing layers of powder to be deposited with the help of a rake or a roller. A conventional laser set-up was used while the build chamber was custom-designed to enable the use of X-ray microtomography at any step of the process, for example immediately after powder deposition or laser melting.

\subsection{Laser set-up}

The laser system used in the present study was developed by the former PHOENIX company, now known as 3D Systems. It consists of a fibre high-energy laser source, a beam expander and a scanning head. The energy is delivered by a $200 \mathrm{~W}$ ytterbium fibre laser from IPG (YLR 4x200W SN). A single head was employed. The laser wavelength is $1080 \mathrm{~nm}$ and the power can be varied continuously from 20 to $200 \mathrm{~W}$. The scanning head includes a motorised galvanometric mirror that controls the final beam motion and position, and an f-theta lens which focuses the laser beam at a distance of roughly $420 \mathrm{~mm}$ from the scanning head. The complete optical path was characterised using a beam analyser from Promotec with spinning blades having calibrated hole sizes. The beam diameter given by the Prolas beam analyser software is defined as the diameter of the spot containing $86 \%$ of the laser power. It was found to be between 50 and $70 \mu \mathrm{m}$ at $\pm 1 \mathrm{~mm}$ from the focal distance.

Safety issues are overcome by interlocking the laser source to the experimental hutch door of the ESRF ID19 beamline. Thus no laser emission is possible when the hutch door is open. 


\subsection{Design of an AM-build chamber adapted for 3D imaging}

The custom-designed build chamber includes a layering system operating in a controlled atmosphere compatible with X-ray microtomography. The developed system consists of a rotating rake and a build plate mounted on a piston that can move up and down along the build direction, see a 3D CAD in Figure 1a. An accurate position of the piston is ensured by a homemade micromechanical device. The position of the build plate is controlled at $\pm 5 \mu \mathrm{m}$ and a maximal displacement rate of $0.5 \mathrm{~mm} / \mathrm{s}$ can be achieved. The build plate mounted on a piston can be moved away from its raking position: up to $10 \mathrm{~mm}$ downwards allowing new layers to be built, and up to $20 \mathrm{~mm}$ upwards to be in a "shadow-free" position to perform in-situ microtomography subsequently to key steps of the layer-by-layer building process. This "shadow-free" position allows microtomography to be performed without missing angles (over 180 or even $360^{\circ}$ if needed). The focal distance of the laser beam was preliminary set to this "shadow-free" position. Thus laser melting and 3D-characterisation are carried out at the same position, i.e. $20 \mathrm{~mm}$ above the layering position.

A rotating rake is used for powder deposition and it also supports the excess powder required to deposit and build additional layers, see an enlarged view of the build chamber interior in Figure 1b. The raking system is confined to the build chamber and consists of a polycarbonate tube, an upper cover made of stainless steel and a bottom plate. The upper cover has a treated window absorbing less than $0.5 \%$ of the laser power. The bottom plate supports the raking system. The build chamber is connected to a vacuum pump and an argon flow and both moving axes are sealed. A controlled atmosphere can thus be employed throughout our experiments. Control of both build plate motion and raking rotation is ensured by dedicated softwares. 


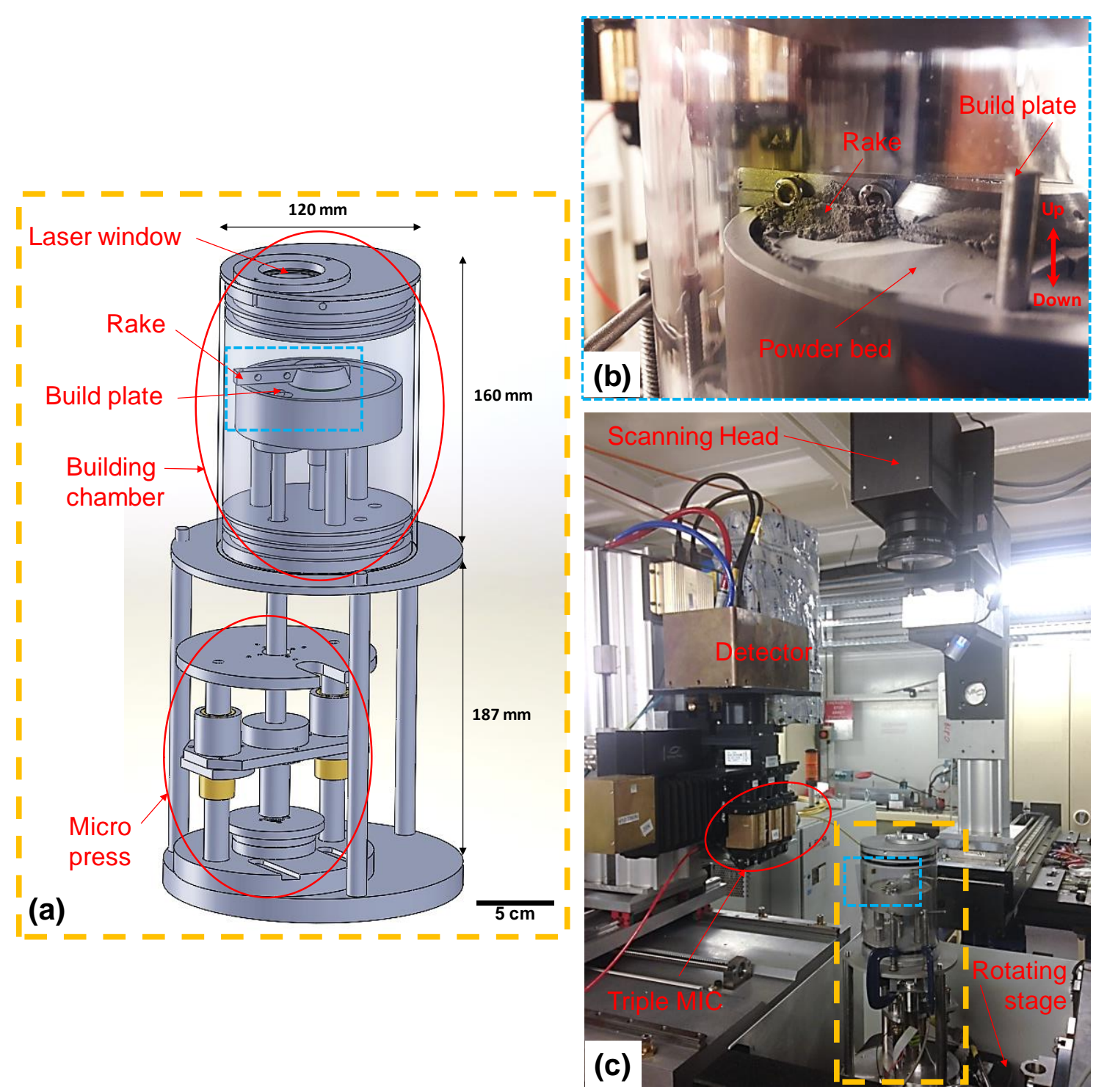

Figure 1. (a) 3D CAD view of the custom-designed build chamber. (b) Enlarged view of the build chamber. (c) Implementation of the AM-system at beamline ID19 of the ESRF.

\subsection{Beamline implementation and imaging conditions}

The whole system consisting of the laser source, scanning head, and build chamber has been implemented at beamline ID19 of the ESRF, see Figure 1c. The laser scanning head is held by a camera manipulator as shown in Figure 1c and mounted on a motorised stage allowing the scanning head to be accurately positioned. Note that the final beam profile analyses (with the whole optical instruments mounted) were performed directly on the rotating stage of the beamline. This enables the laser melting position to be set at the focal distance giving the smallest spot size. The building chamber was placed on the Leuven medium resolution rotating stage. Cables ensuring connection were attached in such a way to allow $180^{\circ}$-rotation of the building chamber.

An indirect X-ray image detector, the so-called TripleMIC (OptiquePeter, Lentilly, France) was used: consisting of a scintillator lens-coupled (Mitutoyo long-working distance objectives) to an sCMOS camera (type: pco.edge 5.5, PCO AG, Germany). Several magnifications can be achieved using a high-energy X-ray beam. Triple-mic enables rapid switching between the 
objectives without the need for focus adjustment and with a scintillator optimised for each magnification. In this feasibility study, magnifications of 2, 5 and 10 times have been tested, resulting respectively in a pixel size of $3.64,1.46$ and $0.73 \mu \mathrm{m}$. However, in this work, image acquisitions were only made with the two-times magnification. Thus, all the reconstructions shown here, have a voxel size of $3.64 \mu \mathrm{m}$. Note that all the images were acquired using the absorption contrast. The scintillator was a $250 \mu \mathrm{m}$ thick LuAg. The experiments were conducted with a ring current of $200 \mathrm{~mA}$. A pink beam was employed using a W150 wiggler with a 40 $\mathrm{mm}$ of gap of filtering with $1.4 \mathrm{~mm}$ of diamond, $1.4 \mathrm{~mm}$ of copper, and $5.6 \mathrm{~mm}$ of aluminium. This results in a peak energy of about $78 \mathrm{keV}$ and a mean integrated detected energy of roughly $95 \mathrm{keV}$. Beam conditions used in this work would also be suitable for heavier metals such as $\mathrm{Ni}$-alloys or steels for a similar building area (build plate diameter of $6 \mathrm{~mm}$ ). The distance between the sample and the detector was about $150 \mathrm{~mm}$ (this can be reduced down to about 100 $\mathrm{mm}$ ). Exposure time was set to $20 \mathrm{~ms} .1500$ projections were acquired per scan resulting in a scan time of $45 \mathrm{~s}$ including reference images. A region of interest (ROI) of 2440 x 1700 pixels which results in a field of view of $8.8 \times 6.2 \mathrm{~mm}$ was used. Data were reconstructed using the ESRF fasttomo3 pre-processing and PyHST2 routines [21] using classical filtered backprojection algorithms. Post-reconstruction ring removal was applied using an in-house ESRFMatlab routine, see [22]. Data were cropped and converted to 8-bits using fixed ROI and grayscale range per sample. Post-processing was conducted using Fiji [23] and in-house plugins [24]. A virtual powder removal procedure has been applied to allow the $3 \mathrm{D}$ rendering of the molten tracks. In other words, the virtual view is a $3 \mathrm{D}$ rendering of the melted wall as it would be observed if the powder bed was removed. Virtual powder removal was performed using image processing and neck detection using Fiji plug-ins. A simple approach based on morphological operators has been employed here: a combination of basic cutoff threshold value determined by iterative intermeans, 3D holes filling, 3D opening (erosion and dilation) using an exact euclidean distance, 3D flood fill, and, classical boolean operations. To summarize, this is roughly equivalent to remove powder particles whose contact surface with the molten track exhibits a radius smaller than $25 \mu \mathrm{m}$ (7 voxels). Note that other images analysis procedures such as watershed or level set based segmentation can be applied without impacting the results.

\subsection{Build procedure}

To run a build with our custom-designed system, the following procedure is applied. An overview of this procedure is given in Figure 2.

First, the build chamber is thoroughly cleaned with a suitable vacuum cleaner and all parts washed with isopropanol. The build plate mounted on the piston is dismantled in order to avoid any contamination with the previously used powder. The build plate can be easily changed to use a similar material than the deposited powder or to use a different material, showing the versatility of our custom-designed build chamber. In the present work, the build plate diameter was $6 \mathrm{~mm}$. Note that the diameter of the build plate can be changed relatively easily. Our system offers the possibility to use build plate with diameters from 4 up to $10 \mathrm{~mm}$. Second, powders of the investigated material are introduced in the build chamber in front of the rake. The initial amount of powder is about a few $\mathrm{cm}^{3}$ to be able to build up multiple layers. Third, the building chamber is closed by placing the polycarbonate tube covered with a stainless steel plate including a window on its support, as shown in Figure 1b. Two O-ring seals are used to simultaneously seal the chamber and hold the polycarbonate tube. Then tubes connecting the building chamber to the vacuum pump and to the argon flow are connected and valves opened. To purge oxygen from the chamber, vacuum pump and argon flow are alternated, repeating 5 
times with a vacuum down to 0.1 mbar. No oxygen sensor was used in the experiments but one will be implemented in a the next-generation building chamber. Valves are finally closed and the vacuum turned off, the building chamber is thus filled with argon. The reference position ("zero") of the build plate mounted on the piston can now be adjusted. This adjustment is carried out by alternately depositing a layer of powder and measuring its thickness using X-ray radiography. To do so, the build plate is moved down in its "raking position" (Figure 2a), the rake is then rotated (Figure 2b) to deposit a powder layer. Depending on the layer thickness, the layering position is adjusted to achieve the desired height. All subsequent layers are then deposited with respect to this position. For instance, the fifth layer will be deposited with the building plate moved down by $500 \mu \mathrm{m}$ for a layer thickness of $100 \mu \mathrm{m}$. The building plate mounted on the piston is then moved up to its "shadow-free" imaging position, see Figure 2cd. A microtomography scan describing the deposited powder bed is acquired and allows the powder bed homogeneity over the region of interest to be assessed. While being at the same imaging position, a laser melting sequence is applied, see Figure 2e (imaging position = melting position). If required, in situ high-speed X-ray radiography can be recorded during laser melting. Any orientation can be selected for registering radiographs. Nevertheless, one should keep in mind that the frame of the scanning head and the X-ray beam source are fixed. Thus if the sample is rotated, one needs to adjust accordingly the laser melting pattern directly in the control software of the laser scanning head. Finally, once the laser melting sequence is completed, a microtomography scan is acquired (Figure 2f). The build plate returns to its layering position and a new powder layer is deposited (Figure 2g). Such a sequence is repeated until the end of the build to obtain a full 3D-description of the build at each layer with images taken after the powder deposition and laser melting steps. We did not observe any motion of the powder particles during the elevation and retraction of the build plate, except on the periphery of the build plate. Typically a region of about $0.5 \mathrm{~mm}$ was affected along the periphery of the build plate. This was assessed by comparing CT-scans acquired during preliminary tests deliberately conducted to detect possible powder particle motion within the powder bed due to elevation and retraction of the build plate. For a dozen of layers built, we did not observe significant changes, this might become an issue for higher builds. However, in such a case, other strategies can be considered. For instance, building a thin wall along the periphery of the build plate.

Our in-situ characterisation presents several advantages over possible ex-situ experiments that deserve to be commented on. (i) Taking out the sample at each processing step might cause oxidation of the built material (powders and molten tracks) induced by drastic atmosphere changes due to interrupted tests and this might affect the building sequence. (ii) Samples would have to be very carefully handled between each layer to limit powder bed stacking changes that could further affect the molten track morphology. For instance, simulations run in Ref [28] demonstrate that the local powder bed density can affect the final molten track morphology. (iii) The positioning of the samples when re-loading the sample into the replicator to ensure a consistent layer thickness throughout the build can be very difficult. (iv) With our in-situ approach, it is possible to make image acquisitions in time corresponding to typical layer processing time without requiring additional time that would likely impact the local thermal conditions. 
(a)

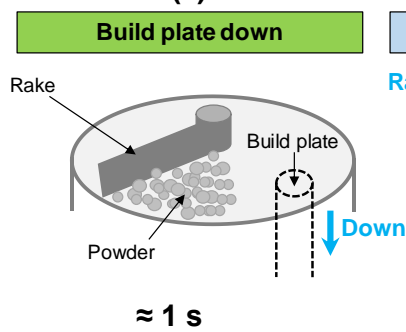

(d)

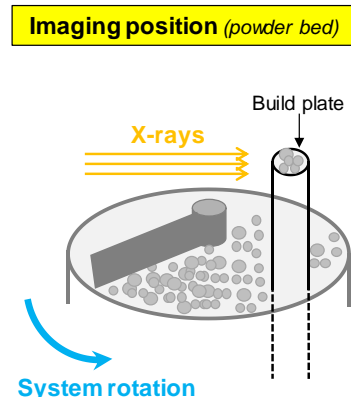

$\approx 45 \mathrm{~s}$ (b)

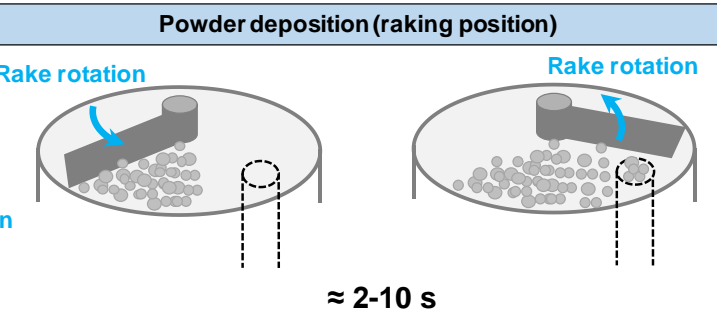

(e)

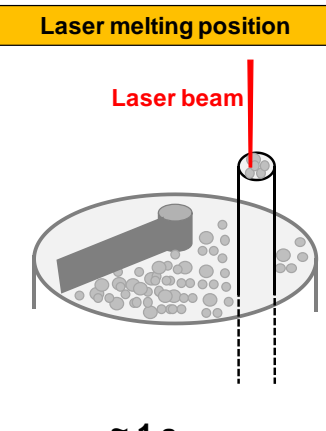

(f)

Imaging position (melted tracks)

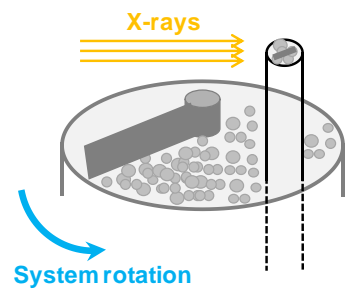

$\approx 45 \mathrm{~s}$ (c)

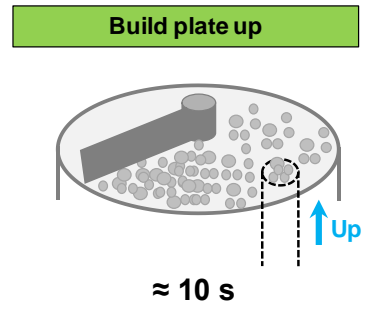

(g)

Build plate down

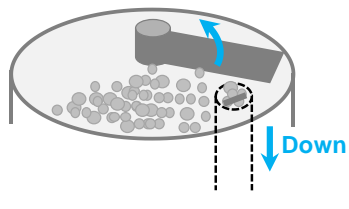

$\approx 10 \mathrm{~s}$

Figure 2. Overview of a build sequence. (a) Build plate down (layer thickness). (b) Rake position: the rake is rotated to deposit powder on top of the build plate. (c) Build plate raised to the imaging position. (d) 3D X-ray imaging to characterise the powder bed. (e) Laser beam melting. (f) 3D X-ray imaging to characterise molten tracks embedded in the powder bed. (g) Build plate lowered for subsequent layer addition. Full characterisation required roughly 2 minutes (time for each step is estimated and indicated).

In the present work, the laser scanning speed and layer thickness were kept constant. The scan speed was set to $200 \mathrm{~mm} / \mathrm{s}$ for all experiments and the building plate was moved 100 $\mu \mathrm{m}$-down at every layer using the micropress depicted in Figure 1a. To vary the linear energy, the laser power was varied between 50 and $200 \mathrm{~W}$. Such parameters were chosen based on a preliminary study that showed that melting tracks with different morphologies can be obtained in this range of power. Objects resembling walls were made of stacks of single melting tracks of $3 \mathrm{~mm}$ in length and were built with varied laser powers and constant speed on the $6 \mathrm{~mm}$ build plate. Walls made of stacked adjacent melting tracks separated by a hatch spacing of $100 \mu \mathrm{m}$ were also built. All of the built objects consisted of multiple layers. In the following, we use a coordinate system $(\mathrm{X}, \mathrm{Y}, \mathrm{Z})$ with $\mathrm{X}$ the scanning direction and $\mathrm{Z}$ the build direction.

\subsection{Material}

The current experiments have been conducted using Ti-6Al-4V-ELI pre-alloyed gasatomized powders, supplied by ARCAM. To facilitate easier powder deposition, a larger powder size distribution, $45-105 \mu \mathrm{m}$, than usually employed in laser-based powder bed fusion machines have been selected. Such a powder size distribution is currently used in AM-electron beam melting machines, another AM-PBF process see e.g. [25-27]. 


\section{Results and Discussion}

\subsection{Building walls made of single melting tracks}

A set of walls made of single tracks were built at constant scan speed $(200 \mathrm{~mm} / \mathrm{s})$ whilst varying the power input: $\mathrm{P} 1=50 \mathrm{~W}, \mathrm{P} 1=100 \mathrm{~W}, \mathrm{P} 3=125 \mathrm{~W}$ and $\mathrm{P} 4=150 \mathrm{~W}$ as indicated in Figure 3. This allows investigation of linear energy $\left(E_{l}\right)$ ranging from 0.250 to $0.750 \mathrm{~J} / \mathrm{mm}$. Figure 3a-f shows 3D renderings of virtual views of the build after the addition of a deposited layer. These virtual views are an original and interesting way to characterise the 3D molten tracks morphology. They are generated using image analysis, in particular, powder neck detections (see details given in section 2.3). The virtual view is a 3D rendering of the melted wall as it would be observed if the powder bed was removed. Note that in our experiments, physical powder removal was only carried out after the final layer was melted. Such virtual views allow the evolution of the morphology of the walls to be monitored as well as the wall internal structure during additive manufacturing (presence of pores). The virtual powder removal routine was not optimised for powder particles close to the building substrate resulting in the presence of few powder particles in contact with the plate as seen in Figure 3a-f.

The evolution of the morphology of the molten tracks can be observed throughout the build from layer \#1 to layer \#6 in Figure 3a-f. After melting the two first layers, one can already detect conditions leading to molten tracks with contrasting morphology. For instance, the single track melted with low power (P1) is discontinuous and shows numerous irregularities as shown in Figure 3a-b. In other words, this condition can be considered typical of unstable melting conditions. This is further exemplified in Figure 4a-b using 2D cross-sections extracted along the melting direction, i.e. in the XZ-plane where partial melting (few powder particles are observed after melting) are evidenced. Interestingly, here the discontinuous molten track does not result from the breakup of a continuous melt pool as typically reported when applying high laser velocities that generate Rayleigh-kind of instabilities (definition of balling), see e.g. [4-6]. Rather, it is thought that droplets already form during melting at low linear energies due to the interplay between wetting, capillary forces and even more importantly the local powder packing/arrangement. In other words, surface tension effects seem greater than the gravity effect resulting in unstable single tracks due to insufficent energy input. Increasing the linear energy leads to the formation of droplets that grow until they reach a constant morphology like those observed for higher linear energies, typically the single tracks melted with P3-P4, see Figure 3a-b. 

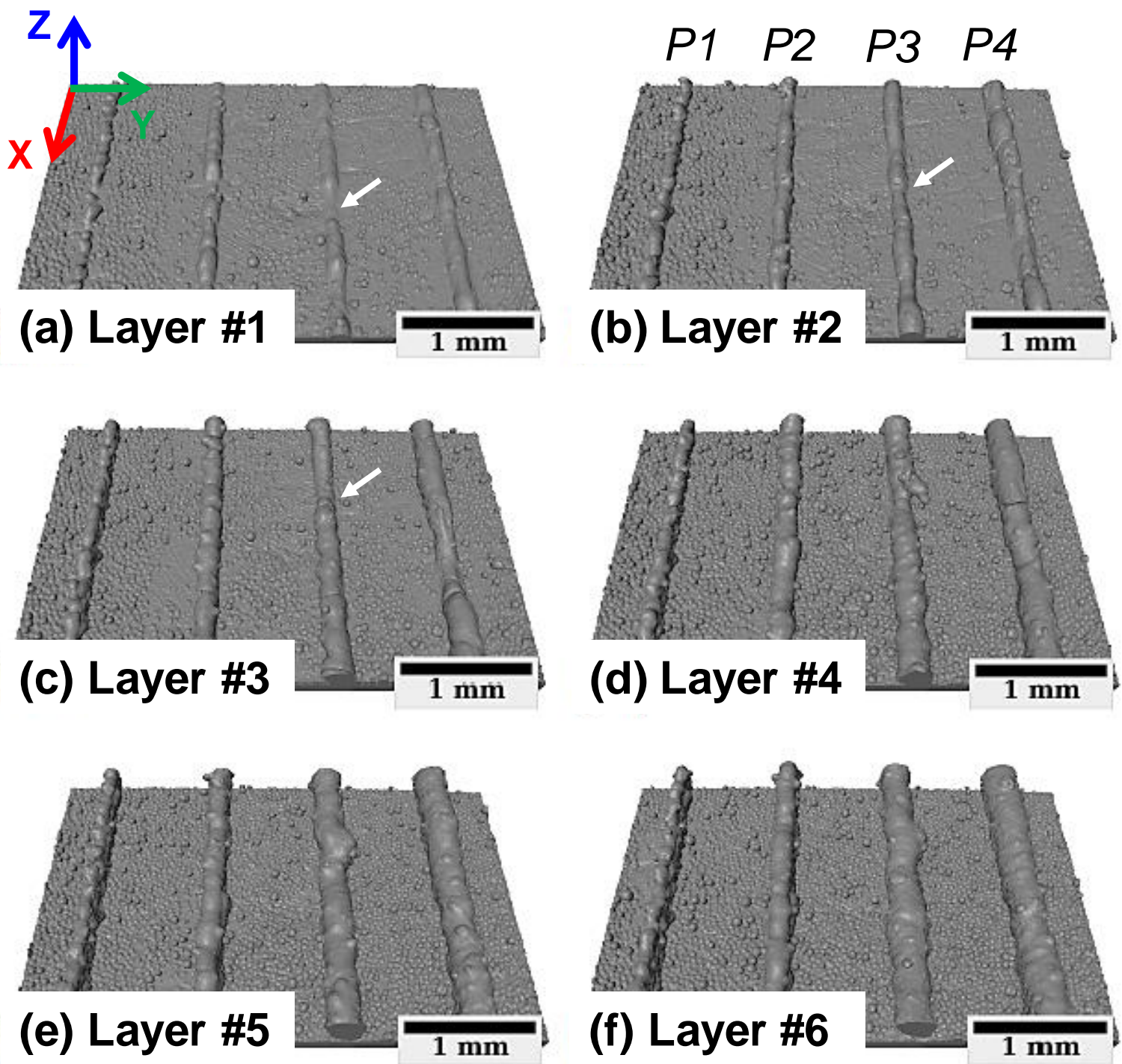

Figure 3. The laser power is increased: $P 1=50 \mathrm{~W}\left(E_{l l}=0.250 \mathrm{~J} / \mathrm{mm}\right), P 2=100 \mathrm{~W}\left(E_{l 2}=0.500 \mathrm{~J} / \mathrm{mm}\right), P 3=125 \mathrm{~W}\left(E_{l 3}=\right.$ $0.625 \mathrm{~J} / \mathrm{mm})$ and $P 4=150 \mathrm{~W}\left(E_{l 4}=0.750 \mathrm{~J} / \mathrm{mm}\right)$ while the laser scanning speed is kept constant $(200 \mathrm{~mm} / \mathrm{s})$ and the layer thickness set to $100 \mu \mathrm{m}$ (defined here as the powder layer height). (a)-(f) 3D view of the walls built by laser additive manufacturing using various laser powers. 3D data have been segmented to virtually remove particles of the powder bed. 


\section{Layer \#1}

(a)

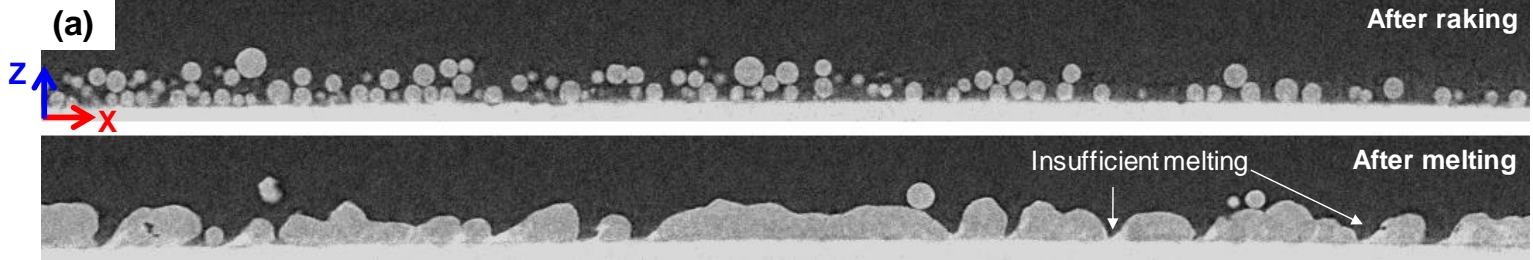

\section{Laver \#2}

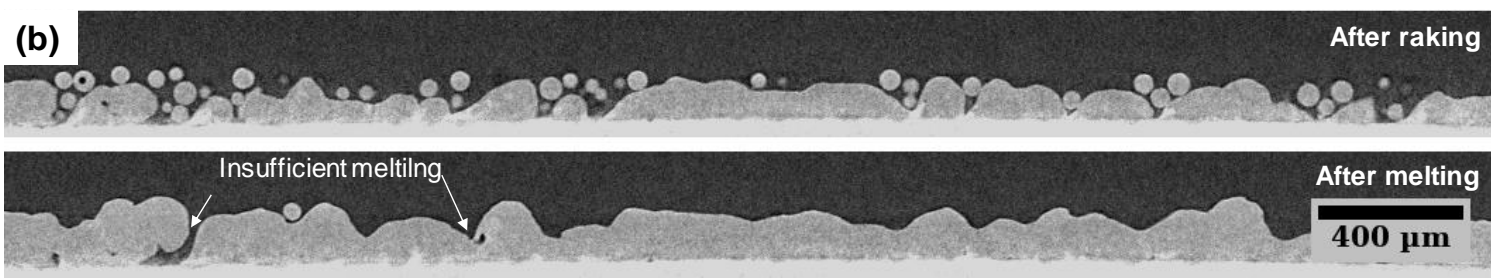

Figure 4. 2D longitudinal cross-sections (X, Z) extracted from the reconstructed volume to illustrate the building sequence of a wall made of single tracks using a power P1 $=50 \mathrm{~W}\left(E_{l 1}=0.250 \mathrm{~J} / \mathrm{mm}\right)$. Two different layers are illustrated (a) and (b) and for each layer we successively show a cross-section after powder deposition (raking) and after laser melting.

The final morphology of the walls made of six different layers and built with increased power is shown in Figure 3f. At low laser power (P1), one can see a thin discontinuous wall with significant height variation whereas at higher power (typically $\mathrm{P} 4$ ) the wall is continuous, larger, and exhibits a mostly smooth surface with a relatively constant height. The average width of the walls increases with the linear energy, see Figure 5, ranging from roughly $150 \mu \mathrm{m}$ for linear energy of $0.250 \mathrm{~J} / \mathrm{mm}$ up to about $350 \mu \mathrm{m}$ at $0.750 \mathrm{~J} / \mathrm{mm}$. Note that for power P1 and P2 the average width of the walls is relatively constant with the addition of new layers whereas the average width of the walls melted using higher power (P3 and P4) increases over the first three layers before achieving a steady-state regime where the width no longer changes between the third and sixth layers. 


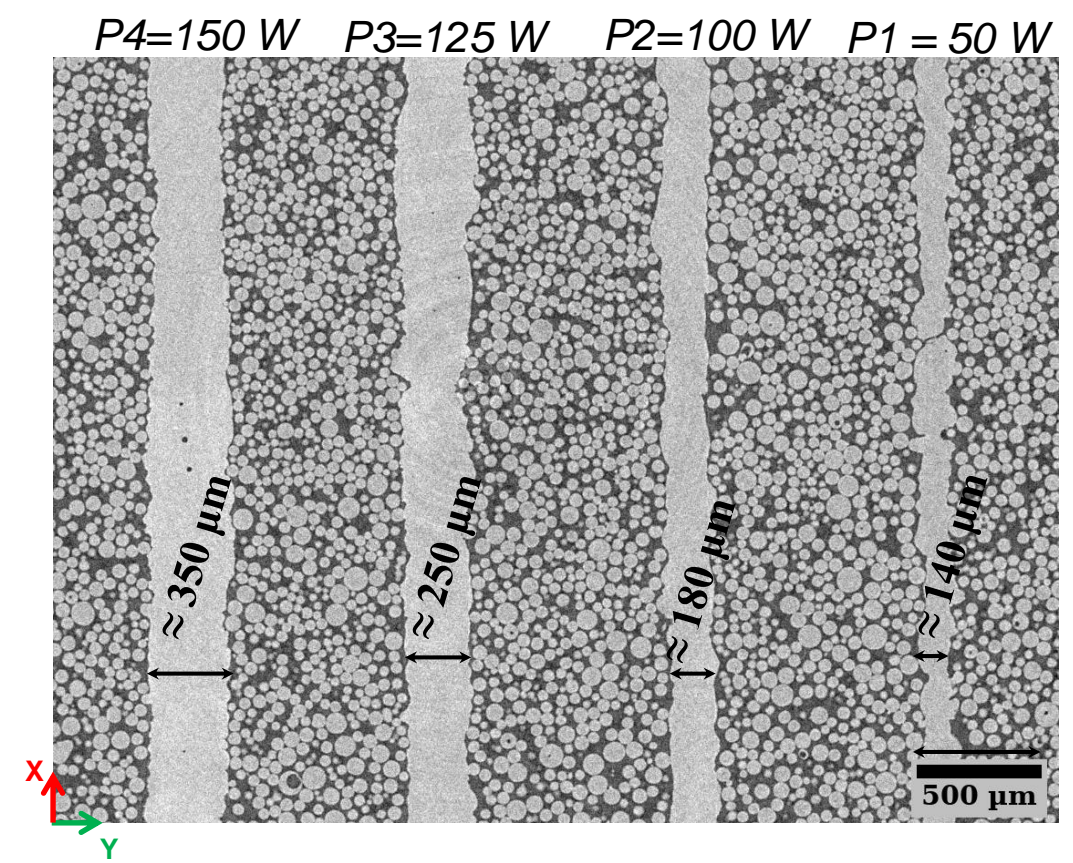

Figure 5. 2D cross-sections parallel to the build plane (X, Y) extracted from the reconstructed volume to illustrate the evolution of the width of the walls made of single tracks with power (layer thickness $=100 \mu \mathrm{m}$ ). This cross-section was extracted at a height corresponding to the addition of layer five.

More interestingly, it is possible to track defect formation and their possible healing by liquid feeding when melting the next layers, thus providing new insights regarding the build history. For instance, in the build history of the track melted with power P4, for layer one, the melting track looks continuous without significant variations of heights (Figure 3b). Subsequently for layer two and layer three, the track P4 shows height variations, see Figure 3c-d, but these tend to be smoothened during the melting of layers four to six, see Figure 3e-f. In addition, the width of the built wall $\mathrm{P} 4$ seems to increase with the addition of new layers. Similarly, the wall built with a power P3 which shows a defect at layer one, indicated by a white arrow in Figure 3b, and which corresponds to a lack of material. This local absence of melted material leads to an irregularity along the melt track that is progressively compensated as new layers are built, see Figure 3c-d. Another kind of defect is generated in the wall P3 at layer four: a protrusion forms (Figure 3d) and is at the origin of the surface irregularity seen in subsequent layers (Figure 3e-f).

Another view of the build sequence when adding new layers is provided by the $2 \mathrm{D}$ transversal cross-sections (Y, Z), see the example shown in Figure 6a-d. These 2D crosssections were extracted from the reconstructed volume at the same position, just after powder deposition as well as after melting, from layer three to layer four. The molten track geometry seems to show a stochastic aspect; this can be seen because the molten track morphology evolves with the addition of a new layer. This indicates that the molten track geometry is sensitive to the local powder arrangement which changes at every layer. This stochastic effect of the molten track geometry can be better exemplified using 3D-imaging, see Figure 7a-h where the build history of a single wall is shown using our virtual views. The latter idea highlights the importance of the local powder stacking, packing, and distribution within the powder bed. The similarity is striking between our experimental results displayed in Figure 6ad with the predictions of the simulation of Korner et al. [28-30] using a 2D Lattice-Boltzmann 
numerical framework [31], see e.g. Figure 6e taken from [29]. Thus our experimental results can be discussed in the light of the large body of modeling work published by Korner and coworkers [28-32]. In reference [28], the effect of the powder bed density on the resulting melt pool shape was investigated. At low relative powder densities $(<50-55 \%)$, it is reported that the melt pool geometry was unstable and did not exhibit a typical hemispherical shape because of the predominance of capillary forces and wetting. In other words, this highlights the key role played by surface tension in comparison with gravity in particular in the presence of a loosely packed powder bed. It was demonstrated that the stabilisation of the melt pool geometry increases with high powder bed densities, typically (>55\%). In order to study the stochastic effect of the powder layer, randomly stacked powder layers were generated while keeping a powder bed density of roughly $55 \%$. Note that in our experiments, 3D measurements of the powder bed relative density gave values within the range of 55-60\% and reveal a rather homogeneous powder bed over the region of interest. It was found that the solidified melt pools strongly depend on the local powder particle arrangement [28]. Those numerical predictions turn out to be consistent with our observations.

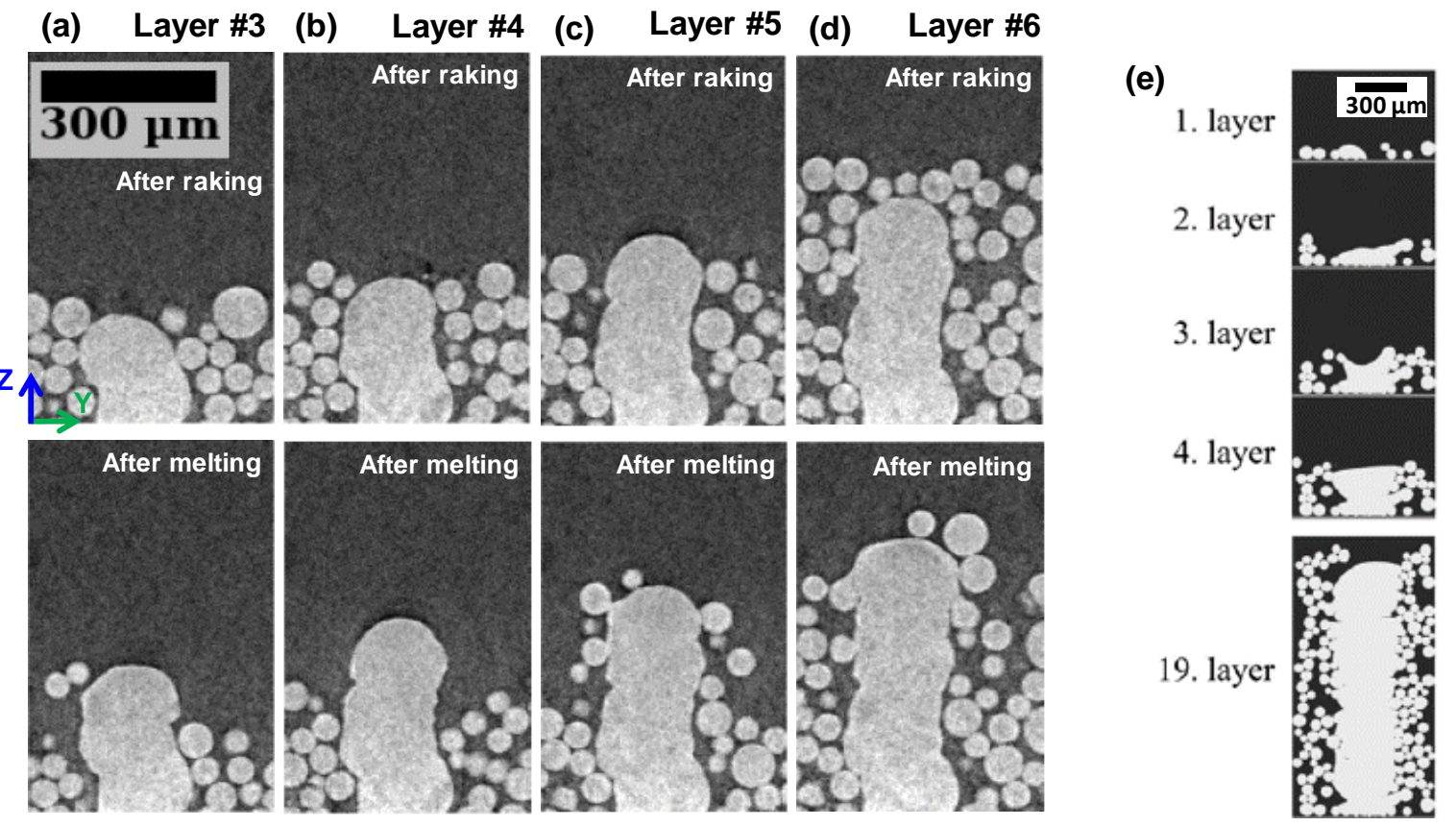

Figure 6. 2D transversal cross-sections $(\mathrm{Y}, \mathrm{Z})$ extracted from the reconstructed volume to illustrate the building sequence of a wall made of single tracks using a power P2 $=100 \mathrm{~W}$ and a speed of $200 \mathrm{~mm} / \mathrm{s}\left(E_{l 2}=0.500 \mathrm{~J} / \mathrm{mm}\right.$ and layer thickness $\left.=100 \mu \mathrm{m}\right)$. Four different layers are illustrated (a)-(d) and for each layer, we successively show a cross-section after powder deposition (raking) and after laser melting. (e) Prediction of the formation of a single wall, extracted from [29] ( $\mathrm{P}=120 \mathrm{~W}, \mathrm{v}=200 \mathrm{~mm} / \mathrm{s}$, $E_{l}=0.6 \mathrm{~J} / \mathrm{mm}$, layer thickness $\left.=100 \mu \mathrm{m}\right)$. 
(a) Layer \#1
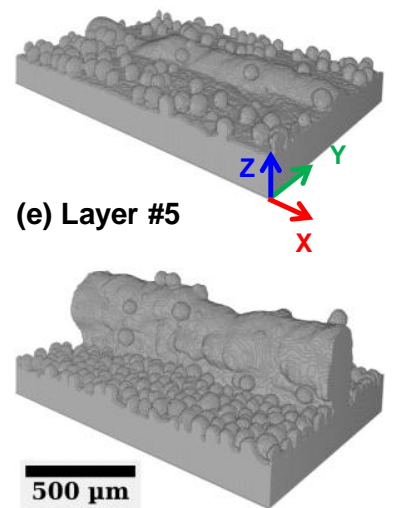

(b) Layer \#2

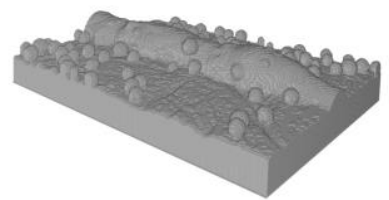

(f) Layer \#6

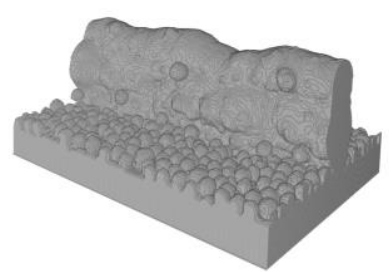

(c) Layer \#3

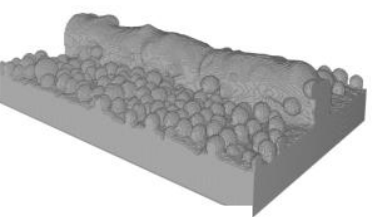

(g) Layer \#7

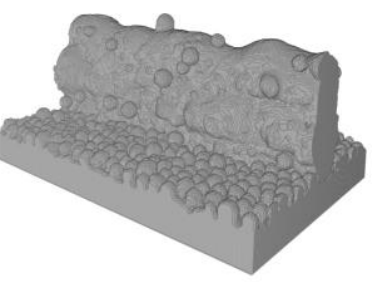

(d) Layer \#4

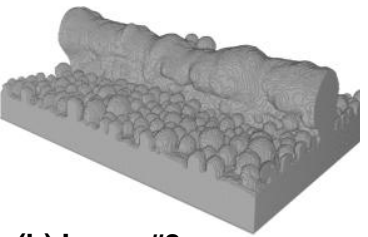

(h) Layer \#8

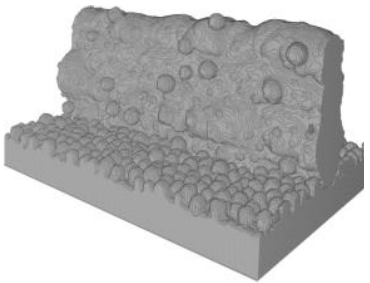

Figure 7. (a)-(h) 3D rendering of the build history of a wall fabricated by laser additive manufacturing using a laser power $\mathrm{P} 2$ $=100 \mathrm{~W}$ (same sample shown in $2 \mathrm{D}$ in Figure 6). 3D data have been segmented to virtually remove particles of the powder bed. The laser scanning speed is kept constant $(200 \mathrm{~mm} / \mathrm{s})$ and the layer thickness set to $100 \mu \mathrm{m}$.

Besides, after melting, we can see that the powder bed height is lower near the melted track than the height of the melted track, meaning that the powder bed is depleted near the melted track. This powder particle depletion in the neighborhood of the single tracks results from powder spattering and is often called the denudation zone, see e.g. [6-8]. The underlying physical mechanisms have been investigated and elucidated by the use of high-speed thermal cameras [7-10] or time-resolved X-ray radiography [12,13,15,17-20]. The denudation zone caused by laser-matter interactions leading to powder spatter is shown when using a larger field of views as illustrated in examples given in Figure 8a-b. After raking, the powder bed height exhibits small variations whereas a clear powder particle depletion near the melted wall is highlighted subsequently to melting events. A schematic illustrating the denudation zone is proposed in Figure 8c for the sake of clarity. Our experimental set-up allows observation of this denudation. Matthews et al. [7] have investigated the width of this denudation zone as a function of the input of the power when melting powders of the same materials, i.e. Ti6Al4V under different atmospheres. For instance, under Ar-pressures of 760 Torr ( 1 bar) and 0.2 Torr $(0.2 \mathrm{mbar})$, the width of the denudation zone was measured to be respectively about $300 \mu \mathrm{m}$ and $600 \mu \mathrm{m}$ for a power of $50 \mathrm{~W}$ and a scan speed of $500 \mathrm{~mm} / \mathrm{s}$. Interestingly, in our case for the same power $(50 \mathrm{~W})$ and a scan speed relatively close $(200 \mathrm{~mm} / \mathrm{s})$, we have estimated the denudation width and we found a width of the same order of magnitude though the powder size distribution $(45-105 \mu \mathrm{m})$ was larger than in [7] (average powder size of $\approx 30 \mu \mathrm{m})$, typically $400 \mu \mathrm{m}$ as shown in Figure 8a-b, demonstrating once again the reliability of our process replicator. 


\section{Layer \#3}

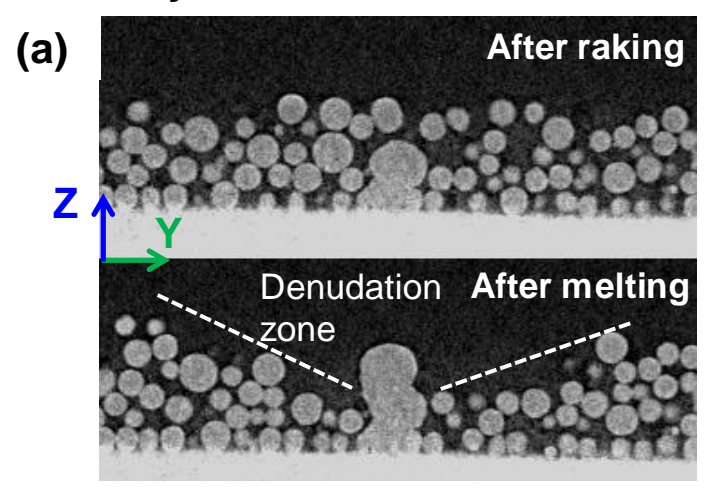

\section{Layer \#4}

(b)
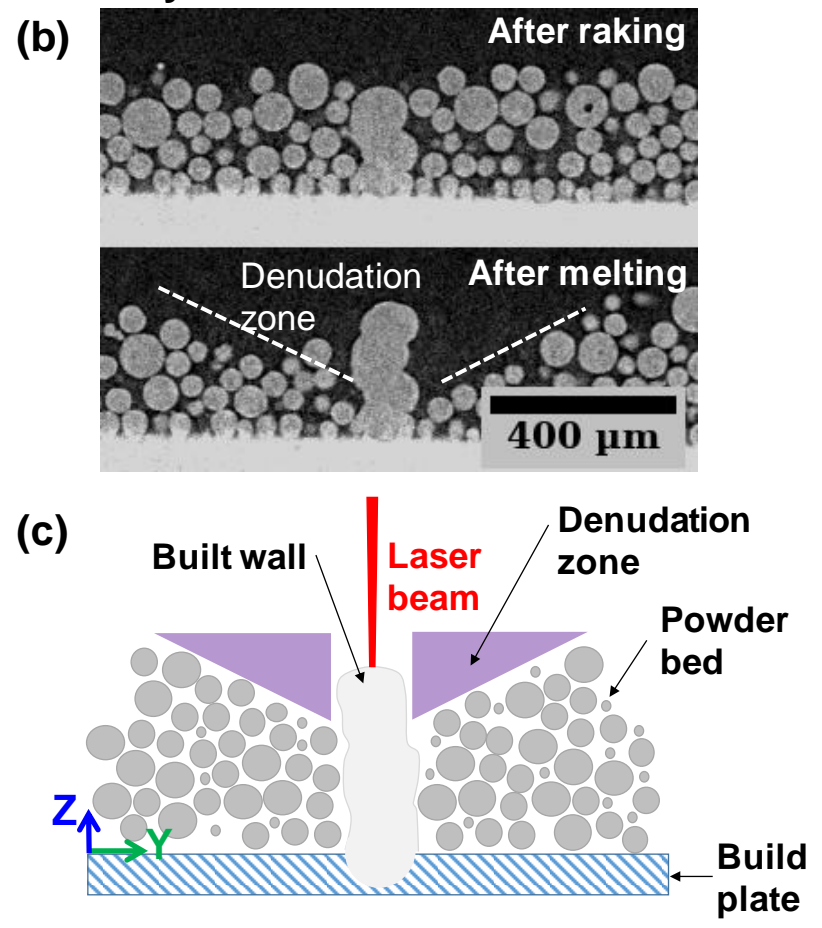

Figure 8. 2D transversal cross-sections $(\mathrm{Y}, \mathrm{Z})$ extracted from the reconstructed volume of the single wall melted with a power $\mathrm{P} 1=50 \mathrm{~W}\left(E_{l l}=0.250 \mathrm{~J} / \mathrm{mm}\right)$ illustrating the presence of a denudation zone. (a) layer one and (b) layer two. We show successive cross-sections after powder deposition and after laser melting. (c) Schematic showing the denudation zone caused by powder spattering.

\subsection{Building walls made of multiple adjacent melted tracks}

AM-parts consists of a large number of adjacent and stacked single tracks. Investigating single melted tracks certainly brings new insights into our fundamental understanding of AMprocesses but it can be seen as an oversimplification in comparison to real 3D-objects. To bring new insights, we built two adjacent melted tracks. Tomographic scans were acquired after powder deposition and after laser melting of every single track. Thus it was possible to monitor evolution of the powder bed and melt pool geometry when two adjacent single tracks were melted.

As a proof-of-concept, we observed the building sequence of the first four layers of the two adjacent single tracks, see Figure 9a-d. Here we have deliberately chosen conditions with low linear energy $(0.250 \mathrm{~J} / \mathrm{mm})$ to favour the formation of defects. For the first layer, it can be seen that the first melted track generates a depleted area, namely the denuded zone as explained 
in Figure 8. Thus fewer powder particles are available to be melted when the laser scans the adjacent line resulting in a difference of height, see Figure 9a. A similar trend is observed for layer two, see Figure 9b. This height difference tends to disappear with the addition of new layers since at layer three and layer four, this height difference is no longer significant. It means that stacking irregularities can be compensated during the process as soon as the parameters are adjusted to ensure a melt pool depth larger than the nominal layer thickness. Indeed, the effective powder layer thickness can be much larger locally than the nominal one. Thus a defect generated at a given layer may no longer exist following the processing of the next layers.

(a)

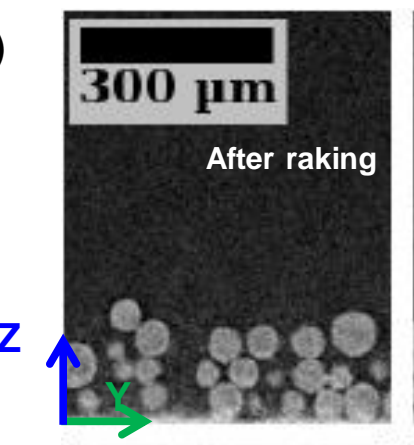

(b)

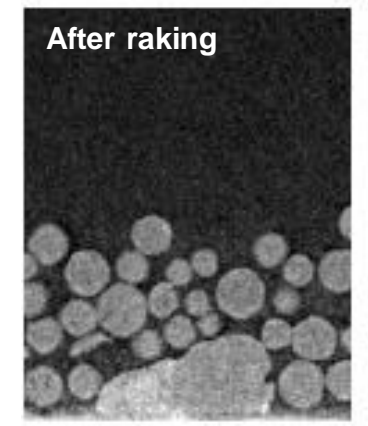

(c)

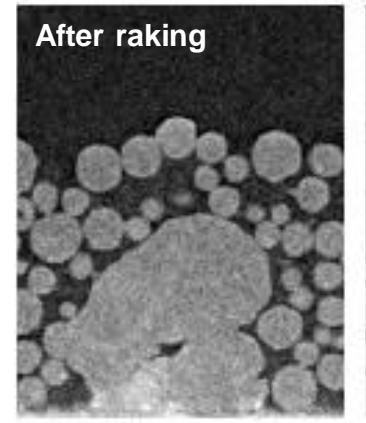

(d)

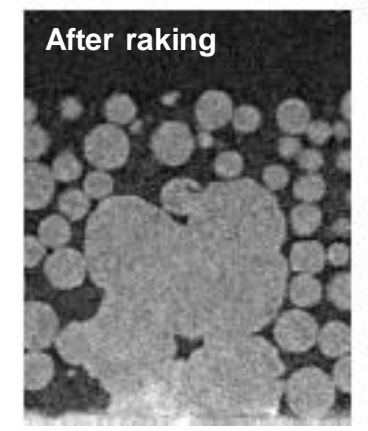

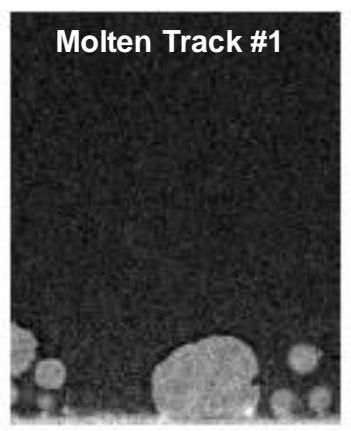
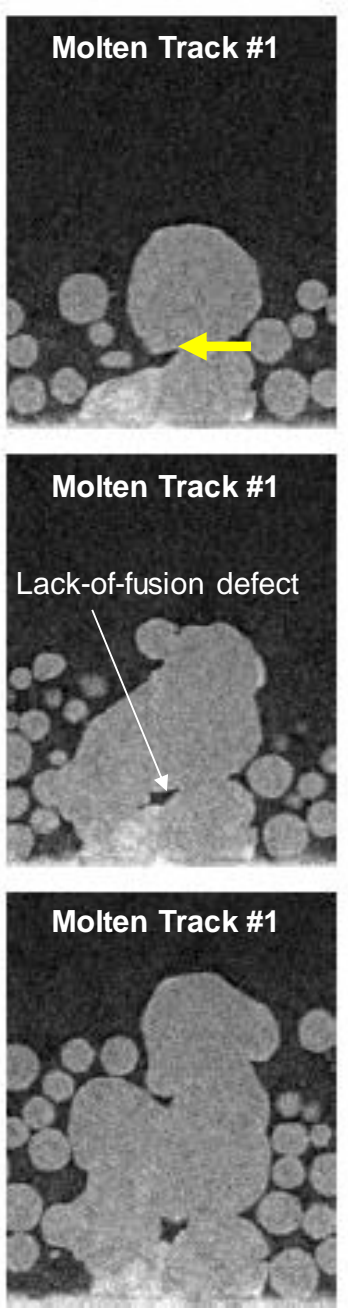

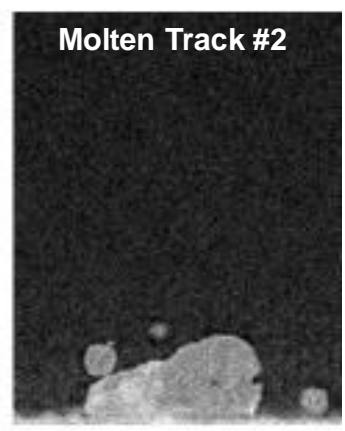

\section{Layer \#1}

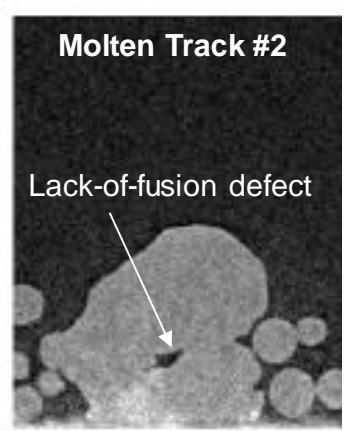

Layer \#2

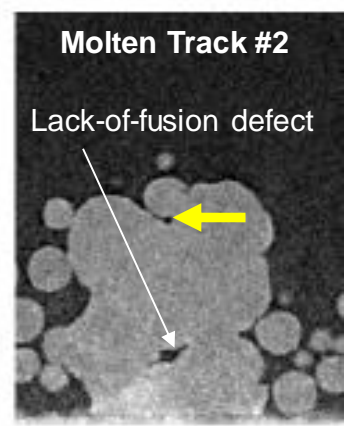

\section{Layer \#3}

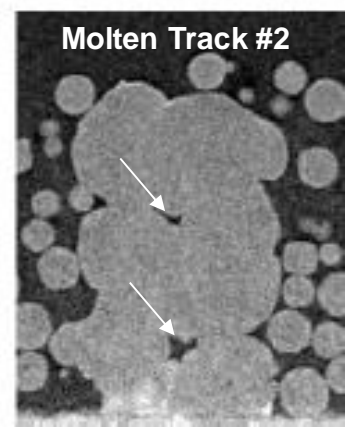

\section{Layer \#4}

Figure 9. Images are acquired once the first track is melted and also subsequently to melting of the adjacent track. Here P1 = $50 \mathrm{~W}\left(E_{l l}=0.250 \mathrm{~J} / \mathrm{mm}\right)$ and the spacing between the two melting track was set to $100 \mu \mathrm{m}$. (a)-(d) $2 \mathrm{D}$ transversal cross-sections $(\mathrm{Y}, \mathrm{Z})$ extracted from the reconstructed volume to illustrate the building sequence of a wall made of two adjacent melting tracks. Four different layers are illustrated and for each layer we successively show a cross-section after powder deposition and after laser melting. 
Figure 9 also reveals another typical defect that has been largely reported in the literature, i.e. the so-called "lack-of-fusion" defect. Here a lack-of-fusion type of defect due to insufficient overlap between the two adjacent melting tracks is detected at the end of layer two once the two adjacent single tracks have solidified, see the defect indicated by a white arrow in Figure 9b). This defect is not healed by liquid feeding during the addition of subsequent layers, hence it is still there after completing layer four (Figure 9d). Interestingly, this defect is caused by a small irregularity which is highlighted by the yellow arrow in Figure 9b. A similar situation seems to occur at the end of layer three where another small irregularity observed on the last solidified layer (see yellow arrow in Figure 9c) is at the origin of the new lack-of-fusion defect revealed in Figure 9d and indicated by the additional white arrow. Again the modelling approach of Bauereiß et al. [32] helps to rationalise the formation of such defects. They demonstrated the minor impact of gravity in comparison with the key role played by the surface tension. Thus liquid feeding of small irregularities when melting the next layer is not necessarily oriented downwards to the underlying layer as one could expect when gravity is predominant. In other words, fluid motion can occur predominantly horizontally preventing the underlying irregularities from being filled by liquid feeding. This results in the formation of a pore, such as the ones shown in Figure 9. Nevertheless, we also found cases where pores were partially healed when melting the next layer. This situation is exemplified in Figure 10a-b where pores $\mathrm{A}$ and $\mathrm{B}$ are partially healed during melting the subsequent layer. The initial large pore $\mathrm{A}$ is now split into two small residual pores while the size of pore B has decreased, unlike pore C which was not affected by the addition of a new layer. One could argue that the healing mechanism could also result from a deep keyhole moving over the lack-of-fusion defect and leaving keyhole pores. However, with the range of melting parameters used in this work, we do not believe that we are in a keyhole regime. Indeed, the inspection of the 3D view of the various molten tracks does not reveal the presence of pores aligned along the molten track at a given depth as highlighted in references [33-35] for melting conditions leading to numerous keyhole pores. This was assessed even for the highest energy input $(P=150 \mathrm{~W}, v=200 \mathrm{~mm} / \mathrm{s})$ expected to be the most sensitive to keyhole porosity.

Those observations rather suggest that we are in a regime governed by heat conduction. Our conclusion is consistent with the results recently published in references [36-38] showing that, with our melting parameters, we should be in a heat conduction mode.

Based on the framework proposed by Patel et al. [37], we have first located our data point on the normalized processing diagrams for L-PBF published in Figure 4 in reference [37]. To do so, the dimensionless energy input, $E^{*}$, and the dimensionless laser velocity, $v^{*}$, were estimated using equations (1) and (2) respectively for all the conditions employed in the present work.

$$
E^{*}=\frac{q^{*}}{l^{*}}
$$

with $\mathrm{q}^{*}=A P / r_{B} \lambda\left(T_{m}-T_{0}\right)$, the dimensionless beam power; and $l^{*}=2 l_{t} / r_{B}$, the dimensionless layer thickness. $A, P, r_{B}, \lambda, T_{m}$ and $T_{0}$ being respectively, the absorptivity, beam power, beam radius, thermal conductivity, liquidus of the investifgated alloy, and powder bed temperature (taken equal to $298 \mathrm{~K}$ here).

$$
v^{*}=\frac{v r_{B}}{\alpha}
$$

with $v$ the laser velocity and $\alpha=\lambda \rho / C p$, the thermal diffusivity; $\rho$ and $C_{p}$ being respectively the density and heat capacity. The physical properties used to run those analytical calculations, and 
extracted from [36], are summarised in Table 1. The results are given in Table 2. Our data points sit within the heat conduction mode region, see Figure 4 in reference [37]. In other words, deep keyholes are not expected.

\begin{tabular}{cccccc}
\hline $\begin{array}{c}\text { Density } \\
\rho\left(\mathrm{kg} / \mathrm{m}^{3}\right)\end{array}$ & $\begin{array}{c}\text { Heat } \\
\text { Capacity } \\
C p\left(\mathrm{~J} \cdot \mathrm{kg}^{-1} \cdot \mathrm{K}^{-}\right. \\
1)\end{array}$ & $\begin{array}{c}\text { Thermal } \\
\text { conductivity } \lambda \\
\left(\mathrm{W} \cdot \mathrm{m}-1 . \mathrm{K}^{-1}\right)\end{array}$ & $\begin{array}{c}\text { Thermal } \\
\text { Diffusivity } \\
\alpha\left(\mathrm{m}^{2} \cdot \mathrm{s}^{-1}\right)\end{array}$ & $\begin{array}{c}\text { Liquidus } \\
\mathrm{T}_{\mathrm{m}}(\mathrm{K})\end{array}$ & $\begin{array}{c}\text { Absorptivity } \\
A\end{array}$ \\
\hline 4430 & 830 & 31,6 & $8.610^{-6}$ & 1923 & 0,6 \\
\hline
\end{tabular}

Table 1. Physcial properties of the Ti-6Al-4V alloy extracted from [36] and used for our analytical calculations of the dimensionless energy input $E^{*}$, dimensionless laser velocity $v^{*}$, and the normalized enthalpy $\Delta H / h_{s}$.

\begin{tabular}{lcccc}
\hline Power, $P(\mathrm{~W})$ & 50 & 100 & 125 & 150 \\
\hline Laser beam velocity, $v(\mathrm{~mm} / \mathrm{s})$ & 200 & 200 & 200 & 200 \\
\hline Linear Energy, $E_{l}(\mathrm{~J} / \mathrm{mm})$ & 0.25 & 0.5 & 0.625 & 0.75 \\
\hline Beam radius $(\mu \mathrm{m})$ & 30 & 30 & 30 & 30 \\
\hline Powder thickness, $l_{t}(\mu \mathrm{m})$ & 100 & 100 & 100 & 58.4 \\
\hline Dimensionless beam power, $q^{*}$ & 19.5 & 38.9 & 48.7 & 0.7 \\
\hline Dimensionless beam velocity, $v^{*}$ & 0.7 & 0.7 & 0.7 & 6.7 \\
\hline Dimensionless layer thickness, $l^{*}$ & 6.7 & 6.7 & 6.7 & 8.8 \\
\hline Dimensionless heat input, $E^{*}$ & 2.9 & 5.8 & 7.3 & 18.8 \\
\hline Normalized enthalpy, $\Delta H / h_{S}$ & 6.2 & 12.5 & 15.7 & evalu \\
\hline
\end{tabular}

Table 2. Summary of the melting conditions used in the present work and results of our calculations to evaluate relevant normalized parameters to determine the dominant melting mode in our experiments.

We have also evaluated the normalized enthalpy using equation (3), as suggested in reference [35] and further applied to Ti-6Al-4V in reference [36] for all the melting conditions used in this work. The results are reported in Table 2.

$$
\frac{\Delta H}{h_{S}}=\frac{A P}{\pi \rho C_{p} T_{m} \sqrt{\alpha v r_{B}}}
$$

The calculated normalized enthalpy reported in Table 2 has been compared to the threshold value for the Ti-6Al-4V alloy. This critical value was found to be about 17 in reference [36]. All values stand below the threshold, except for the highest power which exhibits a value close to the threshold, indicating that our experiments were conducted under a heat conduction mode.

Finally, our experimental observations, as well as analytical calculations based respectively on reference [37] and reference [36] demonstrate that, in this study, we should be in a regime governed by heat conduction. Thus, it is likely that deep keyhole does not form with the melting conditions applied here. As a result, we concluded that the predominant healing mechanism was liquid feeding but not due to deep keyholes. Investigating melting conditions leading to numerous keyhole pores using the characterisation approach described in this work would be worth to be studied in the future. 

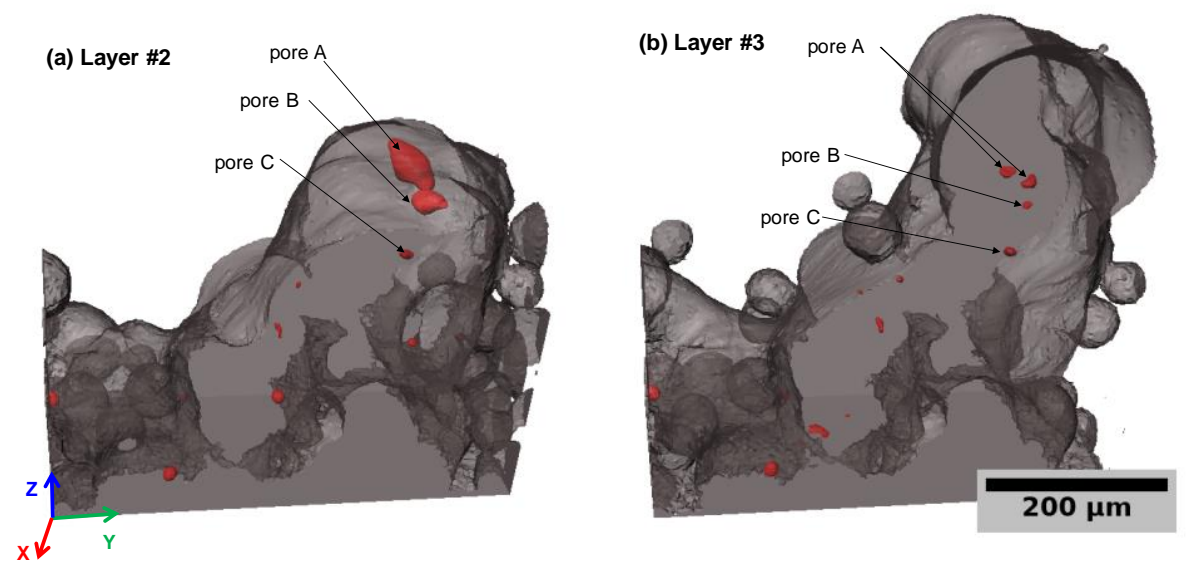

Figure 10. 3D view of a defect observed at layer two (a) and which is partially healed at layer three (b) when building a wall made of two adjacent melting tracks. 3D data have been segmented to virtually remove particles of the powder bed.

In summary, a full 3D non-destructive characterisation was obtained at key steps of the layer-by-layer laser-based powder bed fusion build process. Our approach has provided insights that could not be obtained when performing more classical post-mortem analysis or 2Dcharacterisation. Thus the history of various defects could be investigated in detail. Interestingly, even if numerous defects can be seen when characterising the samples postmortem, in particular when the processing parameters are not optimised as illustrated in Figure 9, a substantial number of defects form during additive manufacturing but disappear when melting adjacent tracks or melting the next layer. This suggests that AM-processes can be seen as inherently self-healing processes. Some of the defects, for example, small lack-of-fusion defects or local surface irregularities along the molten track as typically shown in Figure 3b or Figure 3d, can be healed due to the inherent layer-by-layer building sequence and this can only be revealed and observed using the approach described here.

The results shown in the present work have to be considered as proofs-of-concept rather than a detailed and fundamental investigation of the mechanisms operating when melting a powder bed. However, we demonstrate for the first time that the layer-by-layer sequence inherent to AM-processes can be characterised in 3D through the use of synchrotron X-ray microtomography. Thus our approach turns out to be complementary to the existing tools aiming at characterising in situ AM-powder bed processes such as high-speed optical camera or ultra-fast X-ray radiography. The aim of ongoing work consists in improving our process replicator on two aspects: powder deposition and atmosphere control.

\section{Conclusion}

The main conclusions of the present work can be drawn as follows.

- A custom-design build chamber permitting X-ray microtomography has been designed and implemented at a synchrotron beamline.

- The ability of such an instrument to provide a 3D non-destructive description of the powder bed after steps such as powder deposition or laser melting has been 
demonstrated through a feasibility study. This can be achieved for every layer of a given build or any event of interest.

- Our custom-designed chamber is versatile since we can easily switch from one powder to another.

- The effect of the building strategy on the morphology of melted tracks or the formation of typical defects inherent to AM-processes such as laser-based powder bed fusion can be investigated. Illustrative examples have been provided.

- As a feasibility study, we demonstrate that our approach allows typical situations to be captured such as defect formation and complete or partial healing of the aforementioned defects.

- Such an approach is thought to make the elucidation of fundamental mechanisms possible and to validate existing modelling predictions, but further work is needed to reproduce conditions typical of industrial L-PBF-machines.

- The suggested approach can be used to further investigate and optimise build strategies based on sensitivity to defect formation and spatters. This is the aim of subsequent work.

\begin{abstract}
Aknowledgements
This work was performed within the framework of the Centre of Excellence of Multifunctional Architectured Materials "CEMAM" $\mathrm{n}^{\circ} \mathrm{AN}-10-\mathrm{LABX}-44-01$ funded by the "Investments for the Future Programme', The European Synchrotron Radiation Facility (ESRF) is gratefully acknowledged for offering beamtime associated with proposal MA4066. We thank Edward Mitchell and Gary Adams from ESRF for having edited the manuscript to improve the English style.
\end{abstract}

\title{
References
}

[1] T. Debroy, H.L. Wei, J.S. Zuback, T. Mukherjee, J.W. Elmer, J.O. Milewski, A.M. Beese, A. Wilson-heid, A. De, W. Zhang, Progress in Materials Science Additive manufacturing of metallic components - Process, structure and properties, Prog. Mater. Sci. 92 (2018) 112-224. doi:10.1016/j.pmatsci.2017.10.001.

[2] L.E. Murr, S.M. Gaytan, D.A. Ramirez, E. Martinez, J. Hernandez, K.N. Amato, P.W. Shindo, F.R. Medina, R.B. Wicker, Invited Review and Electron Beam Melting Technologies, Mater. Sci. Technol. 28 (2012) 1-14. doi:10.1016/S10050302(12)60016-4.

[3] S.J. Li, Q.S. Xu, Z. Wang, W.T. Hou, Y.L. Hao, R. Yang, L.E. Murr, Acta Biomaterialia Influence of cell shape on mechanical properties of $\mathrm{Ti}-6 \mathrm{Al}-4 \mathrm{~V}$ meshes fabricated by electron beam melting method, 10 (2014) 4537-4547. doi:10.1016/j.actbio.2014.06.010.

[4] I. Yadroitsev, P. Bertrand, I. Smurov, Parametric analysis of the selective laser melting process, Appl. Surf. Sci. 253 (2007) 8064-8069. doi:10.1016/j.apsusc.2007.02.088.

[5] N.K. Tolochko, S.E. Mozzharov, I.A. Yadroitsev, T. Laoui, L. Froyen, V.I. Titov, M.B. Ignatiev, N.K. Tolochko, S.E. Mozzharov, I.A. Yadroitsev, T. Laoui, L. Froyen, V.I. Titov, M.B. Ignatiev, N.K. Tolochko, S.E. Mozzharov, I.A. Yadroitsev, T. Laoui, L. 
Froyen, V.I. Titov, M.B. Ignatiev, Balling processes during selective laser treatment of powders, Rapid Prototyp. J. 10 (2004) 78-87. doi:10.1108/13552540410526953.

[6] I. Yadroitsev, A. Gusarov, I. Yadroitsava, I. Smurov, Single track formation in selective laser melting of metal powders, J. Mater. Process. Technol. 210 (2010) 16241631. doi:10.1016/j.jmatprotec.2010.05.010.

[7] M.J. Matthews, G. Guss, S.A. Khairallah, A.M. Rubenchik, P.J. Depond, W.E. King, Denudation of metal powder layers in laser powder bed fusion processes, Acta Mater. 114 (2016) 33-42. doi:10.1016/j.actamat.2016.05.017.

[8] S.A. Khairallah, A.T. Anderson, A. Rubenchik, W.E. King, Laser powder-bed fusion additive manufacturing: Physics of complex melt fl ow and formation mechanisms of pores , spatter, and denudation zones, Acta Mater. 108 (2016) 36-45. doi:10.1016/j.actamat.2016.02.014.

[9] V. Gunenthiram, P. Peyre, M. Schneider, M. Dal, F. Coste, R. Fabbro, Analysis of laser - melt pool - powder bed interaction during the selective laser melting of a stainless steel laser melting of a stainless steel, J. Laser Appl. 022303 (2017). doi:10.2351/1.4983259.

[10] V. Gunenthiram, P. Peyre, M. Schneider, M. Dal, F. Coste, I. Koutiri, R. Fabbro, Experimental analysis of spatter generation and melt-pool behavior during the powder bed laser beam melting process, J. Mater. Process. Tech. 251 (2018) 376-386. doi:10.1016/j.jmatprotec.2017.08.012.

[11] A.R. Nassar, M.A. Gundermann, E.W. Reutzel, P. Guerrier, M.H. Krane, M.J. Weldon, Formation processes for large ejecta and interactions with melt pool formation in powder bed fusion additive manufacturing, Sci. Reports. 5038 (2019) 1-11. doi:10.1038/s41598-019-41415-7.

[12] C.L. Alex Leung, S. Marussi, R.C. Atwood, P.D. Lee, M. Towrie, P.J. Withers, In situ X-ray imaging of defect and molten pool dynamics in laser additive manufacturing, Nat. Commun. 9:1355 (2018) 1-9. doi:10.1038/s41467-018-03734-7.

[13] N.D. Parab, L.I. Escano, K. Fezzaa, W. Everhart, A.D. Rollett, L. Chen, T. Sun, Ultrafast X-ray imaging of laser - metal additive manufacturing processes research papers, Jounral Synchrotron Radiat. 25 (2018) 1467-1477. doi:10.1107/S1600577518009554.

[14] N.P. Calta, J. Wang, A.M. Kiss, A.A. Martin, P.J. Depond, G.M. Guss, A.Y. Fong, J.N. Weker, K.H. Stone, C.J. Tassone, J. Matthew, M.F. Toney, A. Van Buuren, M.J. Matthews, N.P. Calta, J. Wang, A.M. Kiss, A.A. Martin, P.J. Depond, G.M. Guss, V. Thampy, A.Y. Fong, J.N. Weker, K.H. Stone, C.J. Tassone, M.J. Kramer, M.F. Toney, A. Van Buuren, M.J. Matthews, An instrument for in situ time-resolved X-ray imaging and diffraction of laser powder bed fusion additive manufacturing processes An instrument for in situ time-resolved X-ray imaging and diffraction of laser powder bed fusion additive manufacturing proce, Rev. Sci. Instrum. 055101 (2018). doi:10.1063/1.5017236.

[15] C. Zhao, K. Fezzaa, R.W. Cunningham, H. Wen, F. De Carlo, L. Chen, A.D. Rollett, T. Sun, Real-time monitoring of laser powder bed fusion process using high-speed X-ray imaging and diffraction, Sci. Rep. 7:3602 (2017) 1-11. doi:10.1038/s41598-01703761-2. 
[16] Q. Guo, C. Zhao, M. Qu, L. Xiong, L.I. Escano, In-situ characterization and quanti fi cation of melt pool variation under constant input energy density in laser powder bed fusion additive manufacturing process, Addit. Manuf. 28 (2019) 600-609. doi:10.1016/j.addma.2019.04.021.

[17] C. Lun, A. Leung, S. Marussi, M. Towrie, R.C. Atwood, P.J. Withers, P.D. Lee, The effect of powder oxidation on defect formation in laser additive manufacturing, Acta Mater. 166 (2019) 294-305. doi:10.1016/j.actamat.2018.12.027.

[18] C. Lun, A. Leung, S. Marussi, M. Towrie, V. Garcia, R.C. Atwood, A.J. Bodey, J.R. Jones, P.J. Withers, P.D. Lee, Laser-matter interactions in additive manufacturing of stainless steel SS316L and 13-93 bioactive glass revealed by in situ X-ray imaging, Addit. Manuf. 24 (2018) 647-657. doi:10.1016/j.addma.2018.08.025.

[19] A.A. Martin, N.P. Calta, J.A. Hammons, S.A. Khairallah, M.H. Nielsen, R.M. Shuttlesworth, N. Sinclair, M.J. Matthews, J.R. Jeffries, T.M. Willey, J.R.I. Lee, Ultrafast dynamics of laser-metal interactions in additive manufacturing alloys captured by in situ X-ray imaging, Mater. Today Adv. 1 (2019) 100002. doi:10.1016/j.mtadv.2019.01.001.

[20] Q. Guo, C. Zhao, L.I. Escano, Z. Young, L. Xiong, K. Fezzaa, W. Everhart, B. Brown, T. Sun, L. Chen, Transient dynamics of powder spattering in laser powder bed fusion additive manufacturing process revealed by in-situ high-speed high- energy x-ray imaging, Acta Mater. 151 (2018) 169-180. doi:10.1016/j.actamat.2018.03.036.

[21] A. Mirone, E. Brun, E. Gouillart, P. Tafforeau, J. Kieffer, Nuclear Instruments and Methods in Physics Research B The PyHST2 hybrid distributed code for high-speed tomographic reconstruction with iterative reconstruction and a priori knowledge capabilities, Nucl. Inst. Methods Phys. Res. B. 324 (2014) 41-48. doi:10.1016/j.nimb.2013.09.030.

[22] A. Lyckegaard, G. Johnson, P. Tafforeau, Correction of ring artifacts in X-ray tomographic images Correction of Ring Artifacts in X-ray Tomographic Images, Int. J. Tomogr. Stat. 18 (2011) 1-9.

[23] C.A. Schneider, W.S. Rasband, K.W. Eliceiri, HISTORICAL commentary NIH Image to ImageJ : 25 years of image analysis, Nat. Methods. 9 (2012) 671-675. doi:10.1038/nmeth.2089.

[24] V. Boulos, V. Fristot, D. Houzet, L. Salvo, P. Lhuissier, V. Boulos, V. Fristot, D. Houzet, L. Salvo, P.L. Investigating, V. Boulos, V. Fristot, D. Houzet, Investigating performance variations of an optimized GPU-ported granulometry algorithm, in: Des. Archit. Signal Image Process., 2012: pp. 1-6.

[25] V. Juechter, T. Scharowsky, R.F. Singer, C. Körner, Processing window and evaporation phenomena for Ti-6Al-4V produced by selective electron beam melting, Acta Mater. 76 (2014) 252-258. doi:10.1016/j.actamat.2014.05.037.

[26] T. Persenot, G. Martin, R. Dendievel, J.Y. Buffiére, E. Maire, Enhancing the tensile properties of EBM as-built thin parts: Effect of HIP and chemical etching, Mater. Charact. 143 (2018) 82-93. doi:10.1016/j.matchar.2018.01.035.

[27] M. Suard, G. Martin, P. Lhuissier, R. Dendievel, F. Vignat, J.J. Blandin, F. Villeneuve, Mechanical equivalent diameter of single struts for the stiffness prediction of lattice structures produced by Electron Beam Melting, Addit. Manuf. 8 (2015) 124-131. 
doi:10.1016/j.addma.2015.10.002.

[28] C. Körner, E. Attar, P. Heinl, Mesoscopic simulation of selective beam melting processes, J. Mater. Process. Technol. 211 (2011) 978-987.

doi:10.1016/j.jmatprotec.2010.12.016.

[29] K. Carolin, M. Markl, A. BauereiB, Fundamental consolidation mechanisms during selective beam melting of powders, Model. And Simulations Mater. Sci. Eng. 21 (2013) 085011. doi:10.1088/0965-0393/21/8/085011.

[30] M. Markl, K. Carolin, Multiscale Modeling of Powder Bed - Based Additive Manufacturing, Annu. Rev. Mater. Res. 46 (2016) 1-31. doi:10.1146/annurev-matsci070115-032158.

[31] R. Ammer, M. Markl, U. Ljungblad, C. Körner, U. Rüde, Simulating fast electron beam melting with a parallel thermal free surface lattice Boltzmann method, Comput. Math. with Appl. 67 (2014) 318-330. doi:10.1016/j.camwa.2013.10.001.

[32] A. Bauereiß, T. Scharowsky, C. Körner, Defect generation and propagation mechanism during additive manufacturing by selective beam melting, J. Mater. Process. Technol. 214 (2014) 2522-2528. doi:10.1016/j.jmatprotec.2014.05.002.

[33] S. Shrestha, T. Starr, K. Chou, A study of Keyhole Porosity in Selective Laser Melting: Single Track Scanning with Micro-CT Analysis, J. of Manuf. Sci. Eng. 141 (2019) 071004. doi:10.1115/1.4043622.

[34] M. Bayat, A. Thanki, S. Mohanty, A. Witvrouw, S. Yang, J. Thorborg, N. Skat, J. Henri, Keyhole-induced porosities in Laser-based Powder Bed Fusion ( L-PBF) of Ti6Al4V : High-fidelity modelling and experimental validation, Addit. Manuf. 30 (2019) 100835. doi:10.1016/j.addma.2019.100835.

[35] W.E. King, H.D. Barth, V.M. Castillo, G.F. Gallegos, J.W. Gibbs, D.E. Hahn, C. Kamath, A.M. Rubenchik, Observation of keyhole-mode laser melting in laser powderbed fusion additive manufacturing, J. Mater. Process. Tech. 214 (2014) 2915-2925. doi:10.1016/j.jmatprotec.2014.06.005.

[36] A.M. Kiss, A.Y. Fong, N.P. Calta, V. Thampy, A.A. Martin, P.J. Depond, J. Wang, M.J. Matthews, R.T. Ott, C.J. Tassone, K.H. Stone, M.J. Kramer, A. Van Buuren, M.F. Toney, J.N. Weker, Laser-Induced Keyhole Defect Dynamics during Metal Additive Manufacturing, Adv Eng. Mater. (2019) 1900455. doi:10.1002/adem.201900455.

[37] S. Patel, M. Vlasea, Melting Modes in Laser Powder Bed Fusion, Materialia 9 (2020) 100591. doi:10.1016/j.mtla.2020.100591.

[38] R. Cunningham, C. Zhao, N. Parab, C. Kantzos, Keyhole threshold and morphology in laser melting revealed by ultrahigh-speed x-ray imaging, Science 852 (2019) 849-852. 